\title{
Una risposta forbita
}

\section{Sara Giovine}

PUBBLICATO: 10 MAGGIO 2019

\section{Quesito:}

Diversi lettori ci scrivono per avere delucidazioni sull'origine e il significato dell'aggettivo forbito. Altri utenti chiedono inoltre quali siano gli altri significati del verbo forbire, oltre a quello letterale di 'pulire, lucidare'.

\section{Una risposta forbita}

a forma forbito, letteralmente 'lucido, terso, nitido' e, in senso figurato, 'curato, raffinato, elegante (riferito specialmente a un discorso, a un modo di parlare o di scrivere, o a uno stile)', rappresenta il participio passato del verbo forbire, che viene però comunemente usato come aggettivo.

Forbire deriva a sua volta dall'antico germanico *furbjan, letteralmente "pulire, lucidare (detto delle armi)', da cui si hanno anche il medio alto tedesco fürben, il francese fourbir, e il provenzale e il catalano forbir, tutti con il significato di 'lustrare, lucidare': il significato originario del verbo, attestato nella nostra lingua a partire dalla seconda metà del XIII secolo, è anche in italiano quello di 'pulire, lucidare, lustrare, restituendo l'originaria lucentezza', con riferimento a oggetti di metallo, stoviglie o cristalli. Si tratta di un significato ampiamente documentato nella storia dell'italiano, con esempi, registrati dal GDLI, da Bono Giamboni, autore del XIII secolo, fino a Corrado Govoni, poeta della prima metà del Novecento:

Che la panziera sua o la catafratta, cervelliera, od elmo sia ben forbito ed ispesso curato, perché molta paura fanno a' nemici l'arme ben forbite e lucenti (Bono Giamboni, Dell'arte della guerra libri IV di Vegezio Flavio).

Un servo sta forbendo il grande specchio / con uno straccio intriso (Corrado Govoni, Poesie).

Tale significato risulta tuttavia oggi ormai raro e alto, come segnalato dalla maggior parte dei dizionari dell'uso, che lo marcano appunto come "non comune" e "letterario".

Al significato specifico di 'lucidare, lustrare un oggetto di metallo (o di altri materiali simili)', si è ben presto aggiunto, per estensione, quello più generico di 'pulire, tergere, asciugare', usato specialmente in riferimento a parti del corpo umano (gli occhi, la fronte, la bocca), anche con il verbo in forma pronominale (forbirsi): nel GDLI se ne rinvengono attestazioni di nuovo in Bono Giamboni e nella Commedia di Dante, nel celeberrimo canto del conte Ugolino, fino ad arrivare a Verga e alla prosa letteraria di Sbarbaro e Soldati:

Come forbirà la sozzura delle mani altrui, colui che porta il loto nelle sue? (Bono Giamboni, Trattati morali, fine XIII secolo).

La bocca sollevò dal fiero pasto / quel peccator forbendola a' capelli / del capo ch'elli avea di retro guasto (Dante, Inf. XXXIII, I-3). 
Disse l'oste forbendosi la bocca col rovescio della manica (Verga, I carbonari della montagna, I86I).

Il contabile che viene a forbire le lenti sulla soglia del fondaco (Sbarbaro, Trucioli, 1920).

Restituì il bicchiere, ringraziò, e si forbì col dorso della mano (Soldati, Le due città, 1964).

Si tratta tuttavia, anche in questo caso, di un significato ormai raro nell'uso corrente, in quanto percepito come più proprio di un registro elevato e letterario.

Raro e letterario, come segnalato dai dizionari sincronici, è oggi anche l'uso figurato della forma riflessiva forbirsi nel significato di 'purificarsi, mondarsi, mantenersi immune da qualcosa tenendosene lontani', di cui si riscontrano attestazioni in italiano da Dante a Pavese:

Gente avara, invidiosa e superba: / Dai lor costumi fa che tu ti forbi (Dante, Inf. XV, 68-69).

Se ti riuscisse di scrivere senza una cancellatura, senza un ritorno, senza un ritocco - ci prenderesti ancora gusto? Il bello è forbirti e prepararti in tutta calma a essere un cristallo (Pavese, Il mestiere di vivere, 1952).

Ugualmente letterario l'uso figurato di forbire per 'raffinare, purificare, perfezionare (lo stile o la lingua)', attestato nella nostra lingua dalla fine del Cinquecento: la prima occorrenza viene rinvenuta dal DELI nel volgarizzamento delle Vite parallele di Plutarco di Marcello Adriani ("Forbi la lingua come strumento da persuadere il popolo con eloquenza ben conveniente e proporzionata al modo del suo vivere"). Tale significato, ancora vitale a fine Ottocento, quando si ritrova, come documentato dal GDLI, nella scrittura critica di De Sanctis e in quella di Carducci, si rivela però oggi, in maniera del resto analoga agli altri significati del verbo, estremamente raro nell'uso corrente:

Nel ciclo cavalleresco vi è una vera progressione: [...] l'uno migliora, l'altro perfeziona; questi forbela forma, quegli lavora sul fondo, finché non giunga Ariosto (De Sanctis, La poesia cavalleresca e scritti vari).

[...] le sue ottave, che più veramente cantano, Catullo ed Orazio le hanno forbite e rimonde da quel superfluo, da quella troppa scorza [...] che impediscono e soffocano più d'una volta la poesia (Carducci, La gioventu di Ludovico Ariosto e la poesia latina in Ferrara).

Una ricerca circoscritta alla sola forma dell'infinito negli archivi moderni dei principali quotidiani nazionali ci conferma la scarsissima diffusione del nostro verbo nella lingua d'uso: i rari esempi riscontrati si possono inoltre motivare con il tentativo di nobilitare la scrittura con il ricorso a un lessico aulico e ricercato (ed è il caso delle prime due occorrenze riportate, tratte rispettivamente dalla recensione di un concerto di Riccardo Muti e da un articolo sul galateo da rispettare per organizzare un perfetto tè pomeridiano) o, viceversa, con la ricerca di effetti ironici, conseguiti proprio attraverso il ricorso a parole ed espressioni desuete o di sapore letterario (è quanto si rileva nel terzo testo, in cui l'autore riflette scherzosamente sul travestimento più adatto in vista di una sua ipotetica partecipazione a una festa in costume organizzata dalla Reggia Reale di Venaria):

Anche dal pianoforte il maestro si preoccupa della concertazione, a volte più che delle note, e se non scatta con direttoriale gestualità della mano libera (fantastico lo slancio per la ripresa del tempo primo nell' Allegro Vivace del Sestetto di Poulenc), l'occhio vigila sul gioco delle parti altrui e ben assestati cenni del capo bastano a forbire l'equilibrio esecutivo, a chiedere un colore solidale o l'intenzione giusta (Angelo Foletto, Il Muti pianista incanta Ravenna, "la Repubblica", 4/7/1997). 
La teiera, da buona protagonista, arriva in tavola per ultima, quando tutti gli ospiti sono già seduti: questi a turno passano la propria tazza (insieme al piattino sottotazza) al padrone di casa, che versa il tè, poi l'appoggiano sul tavolo con accanto il tovagliolino (che useranno sempre piegato per forbire le labbra e le dita), liberando cosi il piattino, su cui mettono i dolci e le tartine di cui via via si servono (Barbara Ronchi della Rocca, Domenica venga a prendere il té da noi, "la Stampa", p. 2I, I/3/1994).

Alla cintola si appendono gli ammennicoli d'oro (o d'acciaio) «atti a forbire gli orecchi, i denti e le unghie», la tabacchiera (dove metterò le medicine da prendere durante la serata), la borsa a rete di seta, dove al posto delle monete troverà posto l'I-phone (mica posso farne a meno). A un rapido controllo mi pare che ci sia tutto, ma come ci arrivo alla Reggia? Conciato così mi lasceranno salire sull'ir che fa capolinea a Venaria? (Bruno Gambarotta, Storie di città, "TorinoSette", 27/9/2013).

Quanto all'aggettivo forbito, analogamente a quanto si è osservato per la voce verbale, il significato originario di 'lucido, terso, netto', usato specialmente in riferimento a oggetti di metallo e ampiamente attestato nella storia dell'italiano letterario a partire dalla metà del XIII secolo (se ne ricordi, fra le altre occorrenze, quella nella celeberrima canzone petrarchesca Chiare, fresche et dolci acque, "qual su le treccie bionde, / choro forbito e perle / Eran quel di a vederle", RVF I26, 46-48), risulta oggi ormai percepito come caratteristico di un registro elevato e letterario ed è di conseguenza di uso piuttosto raro nella lingua corrente.

Più comune l'uso di forbito nel significato figurato di 'curato, raffinato, elegante', con riferimento a un discorso, a un modo di parlare o a uno stile, che si sarebbe diffuso per estensione a partire dal significato originario: il GRADIT lo marca appunto come "comune", mentre gli altri vocabolari si limitano a registrarlo senza ulteriori indicazioni, con l'eccezione del Devoto-Oli 2019, che lo ritiene invece "elevato", e dello Zingarelli 20r9, che lo marca come "parola da salvare". La prima attestazione di tale significato risalirebbe, secondo i dizionari etimologici, all'inizio del Cinquecento, quando compare in un sonetto satirico di Antonio Cammelli ("Ma chi ha sua lingua nel mal dir forbita, / d'invidia è segno e non d'alcuno amore", Rime edite ed inedite), cui seguono numerose altre occorrenze nell'intero arco della storia dell'italiano (il GDLI ne registra esempi fino a Pirandello). L'aggettivo può inoltre assumere valore avverbiale, specialmente allinterno della locuzione parlare forbito, anche in questo caso con attestazioni che giungono a Novecento inoltrato (per esempio in Pratolini "[...] so lasciarmi andare, a momenti. E non sempre, non sempre parlo cosi forbito", Lo scialo, 1960).

Per estensione, forbito può poi riferirsi non solo a un linguaggio o a uno stile, ma anche a una persona, in particolare per indicare 'chi parla o scrive con eleganza e accuratezza formale, in modo ricercato' (per esempio "un oratore forbito"; ma l'aggettivo può essere impiegato anche con la preposizione in, nella costruzione essere forbito nel parlare / nello scrivere) o "chi ha modi compiti e garbati; persona educata, distinta' (per esempio "un giovane forbito / dai modi forbiti").

Si tratta tuttavia di significati che, per quanto non ancora del tutto usciti dall'uso, vengono oggi impiegati prevalentemente in senso ironico, per indicare chi affetta eccessiva eleganza e ricercatezza nel parlare, nello scrivere, o anche nel comportarsi, o per caratterizzare, altrettanto ironicamente, il linguaggio ricercato usato da tali persone: una simile sfumatura ironica si può del resto riconoscere nella maggior parte delle attestazioni dell'aggettivo forbito riscontrate nei quotidiani, di cui si riportano di seguito due esempi.

Riascoltate il breve e, per gli standard di Trump e signora, forbito discorso della vittoria di New York che lo profila rivale della favorita democratica Hillary Clinton. Trump, che inveiva contro Ted Cruz come «Ted il Bugiardo», si rivolge allo sconfitto con rispetto, «il Senatore Cruz» (Gianni Riotta, Metamorfosi Trump: addio a gaffe e insulti per essere presidente, "la Stampa", 21/4/2016). 
Pochi giorni fa in tv il filosofo pop Diego Fusaro - studioso di Antonio Gramsci eppure ideologo del sovranismo nazionalista e identitario - l'ha definita cosi, utilizzando il suo noto forbito e spesso astruso linguaggio: «Vestale del liberalismo precarizzante "no border"» (Matteo Pucciarelli, La seconda vita di Elisa Serafini, l'economia dopo la politica, "la Repubblica", 25/10/2018).

Concludendo, possiamo dire che, se, nell'italiano di oggi, il verbo forbire dà, le pochissime volte che capita di incontrarlo, un'impressione di eccessiva ricercatezza formale o suggerisce un palpabile distanziamento ironico, l'aggettivo derivato forbito, almeno in alcune collocazioni ricorrenti, come oratore forbito, linguaggio forbito e simili, ha ancora qualche modesta circolazione non connotata, anche se neppure dall'uso di questi sintagmi sembra di poter escludere una più o meno marcata ironia sullo stile eccessivamente... forbito di qualcuno.

\section{Cita come:}

Sara Giovine, Una risposta forbita, "Italiano digitale", 2019, IX, 2019/2 (aprile-giugno)

DOI: 10.35948/2532-9006/2020.3113

\section{Copyright 2019 Accademia della Crusca}

Pubblicato con licenza creative commons CC BY-NC-ND 